PREPARED FOR THE U.S. DEPARTMENT OF ENERGY, UNDER CONTRACT DE-AC02-76CH03073

PPPL-3530

PPPL-3530

UC-70

On the Ideal Boundary Condition in a General Toroidal Geometry for a Mixed Magnetic Field Representation

by

X.Z. Tang

December 2000
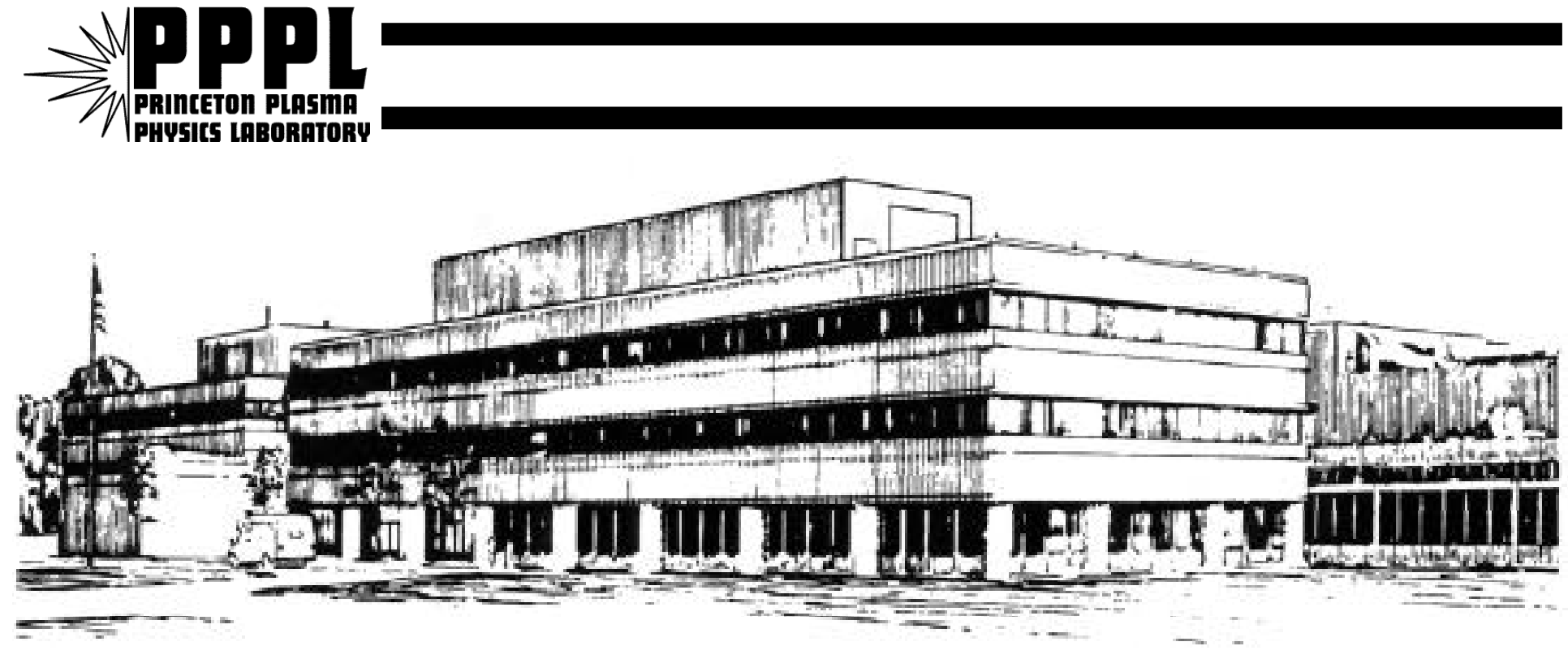

PRINCETON PLASMA PHYSICS LABORATORY PRINCETON UNIVERSITY, PRINCETON, NEW JERSEY 


\section{PPPL Reports Disclaimer}

This report was prepared as an account of work sponsored by an agency of the United States Government. Neither the United States Government nor any agency thereof, nor any of their employees, makes any warranty, express or implied, or assumes any legal liability or responsibility for the accuracy, completeness, or usefulness of any information, apparatus, product, or process disclosed, or represents that its use would not infringe privately owned rights. Reference herein to any specific commercial product, process, or service by trade name, trademark, manufacturer, or otherwise, does not necessarily constitute or imply its endorsement, recommendation, or favoring by the United States Government or any agency thereof. The views and opinions of authors expressed herein do not necessarily state or reflect those of the United States Government or any agency thereof.

\section{Availability}

This report is posted on the U.S. Department of Energy's Princeton Plasma Physics Laboratory Publications and Reports web site in Calendar Year 2000. The home page for PPPL Reports and Publications is: http://www.pppl.gov/pub_report/

DOE and DOE Contractors can obtain copies of this report from:

U.S. Department of Energy

Office of Scientific and Technical Information

DOE Technical Information Services (DTIS)

P.O. Box 62

Oak Ridge, TN 37831

Telephone: (865) 576-8401

Fax: (865) 576-5728

Email: reports@adonis.osti.gov

This report is available to the general public from:

National Technical Information Service

U.S. Department of Commerce

5285 Port Royal Road

Springfield, VA 22161

Telephone: $1-800-553-6847$ or

(703) $605-6000$

Fax: (703) 321-8547

Internet: http://www.ntis.gov/ordering.htm 


\title{
On the ideal boundary condition in a general toroidal geometry for a mixed magnetic field representation
}

\author{
X. Z. Tang* \\ Princeton Plasma Physics Laboratory, Princeton University, Princeton, NJ 08543
}

(January 3,2000 )

\begin{abstract}
Subtleties of implementing the standard perfectly conducting wall boundary condition in a general toroidal geometry are clarified for a mixed scalar magnetic field representation. An iterative scheme based on Ohm's law is given.
\end{abstract}

*Email: xtang@pppl.gov 


\section{INTRODUCTION}

Boundary conditions can play a non-trivial role in the solution of the partial differential equations [1] arising from the continuum modeling of fusion plasmas [2,3]. One example is the perfectly conducting wall, or the ideal, boundary condition for nonlinear resistive magneto-hydrodynamics (MHD) simulations. A perfectly conducting wall implies the normal magnetic field and the parallel electric field both vanish at the boundary. This can be trivially implemented if the resistive MHD equations are solved in the primitive variables $\mathbf{B}$ and $\mathbf{E}$. In practice, however, this is often not the case due to other considerations. For example, MH3D [2] has employed a mixed scalar representation for the magnetic field, equation (1),

$$
\mathbf{B}=\nabla \psi \times \nabla \varphi+\frac{1}{R} \nabla_{\perp} F+\frac{R_{0}}{R} I \hat{\varphi}
$$

in order to decouple the fast, but stable, compressional wave from the shear Alfvén wave. $F$ and $I$ are not independent, and are related by the divergence-free constraint for $\mathbf{B}$ in the form of a Poisson equation for $F$ with $I$ an effective source term.

Using Faraday's law, we show that axisymmetry presents an unusual and fragile situation that $\psi=0$ and $\partial F / \partial n=0$ satisfy the three ideal boundary constraints simultaneously. The breaking of the axisymmetry convolutes the primitive variables enough that the boundary condition can only be satisfied iteratively. The formulation based on Faraday's law becomes fatally flawed if the normal of the outer most magnetic surface is perpendicular to the toroidal direction anywhere on the boundary, $\hat{\mathbf{n}} \cdot \nabla \varphi=0$.

We propose an alternate formulation based on Ohm's law. Ideal boundary condition implies that the tangential plasma current vanishes on the boundary of a resistive plasma. The poloidal component of the tangential current is found to provide an inhomogeneous Neumann boundary condition for the toroidal field evolution, equation (22). The toroidal

component of the tangential current naturally gives rise to an inhomogeneous Dirichlet condition for evolving the Laplacian of $\psi$, equation (24). The flux function is inverted from its Laplacian with a homogeneous Dirichlet boundary condition, equation (18). This 
formulation is well defined for arbitrary toroidal geometry and makes a smooth transition if the configuration is axisymmetric. It also clarifies that the optimal strategy to study magnetic evolution is to follow the toroidal field $I$ and the dominant component of the toroidal current $\Delta^{\star} \psi \equiv \nabla_{\perp}^{2} \psi-\frac{1}{R} \frac{\partial \psi}{\partial R}$.

The rest of the paper is organized as follows. In section II we introduce the mixed scalar representation of the magnetic field and discuss the general boundary condition requirement associated with resistive MHD. The subtleties of implementing the ideal boundary condition in a general toroidal geometry are clarified in section III using Faraday's law. An alternate, improved, formulation based on Ohm's law is given in section IV.

\section{SCALAR MAGNETIC FIELD REPRESENTATION AND MHD}

Three dimensional MHD codes such as MH3D use a mixed poloidal flux and toroidal field representation for the magnetic field. This can be seen by writing the vector potential into toroidal and poloidal plane components

$$
\mathbf{A}=\nabla_{\perp}\left(f+f_{0}\right) \times \hat{\varphi}+\psi \nabla \varphi
$$

in the standard $(R, Z, \varphi)$ cylindrical coordinates with $\varphi$ the toroidal angle. Here $\nabla_{\perp} \equiv$

$\hat{\mathbf{R}} \frac{\partial}{\partial R}+\hat{\mathbf{Z}} \frac{\partial}{\partial Z}$ is a gradient in the poloidal plane. Taking the curl of the vector potential, one finds the magnetic field of the form

$$
\mathbf{B}=\nabla \psi \times \nabla \varphi+\frac{1}{R} \nabla_{\perp} F+\frac{R_{0}}{R} I \hat{\varphi}
$$

with $F \equiv \frac{\partial f}{\partial \varphi}$ and $R_{0}$ the major radius. The introduction of $f_{0}(R, Z)$ in the vector potential is to separate out the strong axisymmetric toroidal field, for the total toroidal field is

$$
\frac{R_{0}}{R} I=-\nabla_{\perp}^{2} f-\nabla_{\perp}^{2} f_{0}
$$

The standard choice for $f_{0}$ is based on the single wire model,

$$
-\nabla_{\perp}^{2} f_{0}(R)=\frac{R_{0}}{R} B_{\varphi_{0}}
$$


In dimensionless form (scale $\mathbf{B}$ by $B_{\varphi_{0}}$ ),

$$
I=1+\epsilon \tilde{I} \text { with } \tilde{I} \equiv-\frac{R}{a} \nabla_{\perp}^{2} f
$$

The inverse aspect ratio is $\epsilon \equiv a / R_{0}$ and $a$ is the minor radius.

The standard MHD algorithm is to solve $\psi$ and $\tilde{I}$ from the magnetic induction equation, and then finds $f$ from

$$
\nabla_{\perp}^{2} f=-\frac{a}{R} \tilde{I}
$$

a condition required to enforce divergence-free of the magnetic field.

For resistive MHD, the dissipative term $\eta \nabla^{2} \mathbf{B}$ in the induction equation, which is of second order, requires boundary conditions for a proper solution. Altogether, the induction equation and diverge-free constraint demand three separate boundary conditions. These are exactly satisfied by the ideal boundary conditions of vanishing normal magnetic field and tangential electric fields.

There is freedom in converting the vector induction equation into scalar equations, especially for $\psi$. The original spectral MH3D solves the time evolutions for $\tilde{I}$ and $\psi$ of the form

$$
\begin{aligned}
& \frac{\partial \tilde{I}}{\partial t}=\text { lower order terms }+\eta \nabla_{\perp}^{2} \tilde{I} \\
& \frac{\partial \psi}{\partial t}=\text { lower order terms }+\eta \nabla_{\perp}^{2} \psi
\end{aligned}
$$

By lower order terms we have actually included those second order toroidal derivative terms that are treated explicitly and require no boundary condition (periodic, to be precise). The later version of MH3D, which uses real representation for field variables, chooses to follow the time evolution of $\nabla_{\perp}^{2} \psi$ directly for numerical accuracy considerations of finite element representation. The equation is of a familiar form

$$
\frac{\partial}{\partial t} \nabla_{\perp}^{2} \psi=\text { lower order terms }+\nabla_{\perp}^{2}\left(\eta \nabla_{\perp}^{2} \psi\right) \text {. }
$$

A boundary condition for $\nabla_{\perp}^{2} \psi$ is required in this case. 


\section{SUBTLETIES OF BOUNDARY CONDITION IMPLEMENTATION IN A GENERAL TOROIDAL GEOMETRY}

\section{A. General consideration using Faraday's law}

For a perfectly conducting wall, the E\&M boundary condition is

$$
\left.\mathbf{B} \cdot \hat{n}\right|_{b d y}=0 \text { and } \hat{n} \times\left.\mathbf{E}\right|_{b d y}=0 .
$$

The electric potential $\Phi$ is a constant on the wall, which is usually set to be zero

$$
\left.\Phi\right|_{b d y}=0
$$

The wall is rigid so no plasma can flow out

$$
\left.\mathbf{v} \cdot \hat{n}\right|_{b d y}=0
$$

Translating these physical boundary condition into constraints for the scalar variables can be problematic. To illustrate the subtleties involved, let's introduce a new set of, VMEC-

like, coordinates $(s, \theta, \varphi)$ [4]. The $s$ is an equilibrium toroidal magnetic flux label, $s$ being one on the outer-most magnetic surface (where the ideal wall resides) and vanishing at the equilibrium magnetic axis. We have assumed that the reference equilibrium is made of closed magnetic surfaces. The $\theta$ is a poloidal angle, which can be the VMEC poloidal angle, or a magnetic one. The toroidal angle $\varphi$ is a geometrical one, coincides with the cylindrical coordinates.

The magnetic field is

$$
\mathbf{B}=\nabla \psi \times \nabla \varphi+\frac{1}{R} \nabla_{\perp} F+R_{0} I \nabla \varphi
$$

The normal component of the field can be found, with a scaling factor, by dotting $\mathbf{B}$ with $\nabla s$,

$$
\mathbf{B} \cdot \nabla s=\nabla \psi \times \nabla \varphi \cdot \nabla s+\frac{1}{R} \nabla_{\perp} F \cdot \nabla s+R_{0} I \nabla \varphi \cdot \nabla s .
$$


Setting it to be zero, one has

$$
\nabla \psi \times \nabla \varphi \cdot \nabla s+\frac{1}{R} \nabla_{\perp} F \cdot \nabla s+R_{0} I \nabla \varphi \cdot \nabla s=0 .
$$

The form of this equation implies that it can either serve as an inhomogeneous Dirichlet boundary condition for $I$ or as an inhomogeneous Neumann boundary condition for $F$.

The boundary condition on the tangential electric field can be straightforwardly applied on Faraday's law. Faraday's law can be written as

$$
\frac{\partial \mathbf{A}}{\partial t}=-\mathbf{E}+\nabla \Phi
$$

with

$$
\mathbf{A}=\nabla_{\perp}\left(f+f_{0}\right) \times R \nabla \varphi+\psi \nabla \varphi
$$

Applying $\nabla s \times$ on this equation, one has

$$
\frac{\partial}{\partial t} \nabla s \times \mathbf{A}=-\nabla s \times \mathbf{E}+\nabla s \times \nabla \Phi .
$$

On the boundary, both $\nabla s \times \mathbf{E}$ and $\nabla s \times \nabla \Phi$ vanish, so

$$
\nabla s \times\left.\mathbf{A}\right|_{b d y}=\nabla s \times\left.\mathbf{A}_{0}\right|_{b d y},
$$

with $\mathbf{A}_{0}$ the initial vector potential. This vector equation can be broken into poloidal and toroidal components by dotting it with $\nabla \theta$ and $\nabla \varphi$.

$$
\begin{aligned}
& \nabla \theta \cdot \nabla s \times\left.\mathbf{A}\right|_{b d y}=\nabla \theta \cdot \nabla s \times\left.\mathbf{A}_{0}\right|_{b d y} \\
& \nabla \varphi \cdot \nabla s \times\left.\mathbf{A}\right|_{b d y}=\nabla \varphi \cdot \nabla s \times\left.\mathbf{A}_{0}\right|_{b d y}
\end{aligned}
$$

The result is

$$
\begin{aligned}
\nabla \theta \cdot \nabla s \times \mathbf{A}= & \nabla \theta \cdot \nabla s \times\left[\nabla_{\perp}\left(f+f_{0}\right) \times R \nabla \varphi\right]+\nabla \theta \cdot \nabla s \times(\psi \nabla \varphi) \\
= & R(\nabla s \cdot \nabla \varphi)\left[\nabla_{\perp}\left(f+f_{0}\right) \cdot \nabla \theta\right]-R\left[\nabla s \cdot \nabla_{\perp}\left(f+f_{0}\right)\right](\nabla \theta \cdot \nabla \varphi) \\
& +(\nabla \theta \cdot \nabla s \times \nabla \varphi) \psi \\
\nabla \varphi \cdot \nabla s \times \mathbf{A}= & \nabla \varphi \cdot \nabla s \times\left[\nabla_{\perp}\left(f+f_{0}\right) \times R \nabla \varphi\right]+\nabla \varphi \cdot \nabla s \times(\psi \nabla \varphi) \\
= & -\frac{1}{R} \nabla s \cdot \nabla_{\perp}\left(f+f_{0}\right)
\end{aligned}
$$


Hence the E\&M boundary condition for a three dimensional device is

$$
\begin{array}{r}
\nabla \psi \times \nabla \varphi \cdot \nabla s+\frac{1}{R} \nabla_{\perp} F \cdot \nabla s+R_{0}(1+\epsilon \tilde{I}) \nabla \varphi \cdot \nabla s=0 \\
R(\nabla s \cdot \nabla \varphi)\left(\nabla_{\perp} f \cdot \nabla \theta\right)-R\left(\nabla s \cdot \nabla_{\perp} f\right)(\nabla \theta \cdot \nabla \varphi)+(\nabla \theta \cdot \nabla s \times \nabla \varphi) \psi=C_{\psi} \\
\nabla s \cdot \nabla_{\perp} f=C_{f}
\end{array}
$$

where $C_{\psi}$ and $C_{f}$ are time independent functions set by the initial condition.

\section{B. Axisymmetric configuration}

Axisymmetric toroidal configuration, like that of a Tokamak, simplifies the boundary constraints remarkably, and that is also where numerical computation had its greatest success $[2,3]$. Axisymmetry implies $\nabla \varphi \cdot \nabla s$ identically vanishes. The most important consequence is that equations $(10,11,12)$ become over-determined,

$$
\begin{aligned}
\nabla \psi \times \nabla \varphi \cdot \nabla s+\frac{1}{R} \nabla_{\perp} F \cdot \nabla s & =0 ; \\
-R\left(\nabla s \cdot \nabla_{\perp} f\right)(\nabla \theta \cdot \nabla \varphi)+(\nabla \theta \cdot \nabla s \times \nabla \varphi) \psi & =C_{\psi} ; \\
\nabla s \cdot \nabla_{\perp} f & =C_{f} .
\end{aligned}
$$

The choice of

$$
\psi=0 \text { and } \nabla s \cdot \nabla_{\perp} F=0
$$

would satisfy the three constraints of the ideal boundary condition simultaneously. We note that $\nabla s \cdot \nabla_{\perp} F=0$ implies $\nabla s \cdot \nabla_{\perp} f=C_{f}(R, Z)$ since $F \equiv \partial f / \partial \varphi$. Equation (16) is nothing but a homogeneous Dirichlet condition for $\psi$ and a homogeneous Neumann condition for $F$. Despite this simplicity, the boundary condition for $\tilde{I}$ is undetermined from this derivation. In earlier practice [5], the time evolution equation for $\tilde{I}$ is supplemented by an inhomogeneous Dirichlet condition $\tilde{I}=\tilde{I}_{0}$ if $\eta$ is finite, or a homogeneous Neumann boundary condition if $\eta$ vanishes on the edge. 


\section{Non-axisymmetric configuration}

Non-Axisymmetry, in principle, removes the over-determinedness or ill-poseness in the axisymmetric case, of the boundary constraints given in equations $(10,11,12)$. In reality, the possibility of $\nabla s \cdot \nabla \varphi \neq 0$ makes the matter much trickier. Due to the convoluted nature of the boundary condition in our scalar variables, it is impossible to satisfy all of them simultaneously while decoupling the various degrees of freedom (means solving equations separately). An iterative approach is required, resembling that of feedback control. In addition, reconstructing an initial profile in our scalar variables from other equilibrium solvers, would require special consideration for satisfying the boundary conditions.

A straightforward iterative scheme to satisfy equations $(10,11,12)$ within a time step is as follows. One first applies equation (12) as an inhomogeneous Neumann boundary condition to find $f$ given $\tilde{I}$ at previous time step. The obtained $f$ is then substituted into equation (11) and turns this equation into an inhomogeneous Dirichlet condition to advance $\psi$. With the knowledge of $\psi$ and $f(F)$, equation (10) provides an inhomogeneous Dirichlet condition to advance $\tilde{I}$. In principle, the new $\tilde{I}$ can be used as a new source in the Poisson equation for $f$ to iterate the $f, \psi$, and $\tilde{I}$ to desired accuracy within one time step. This is probably not necessary in practice, since the wall mode is of considerable longer time scale than the time step that is limited by the Courant-Friedrichs-Lewy condition of the shear Alfvén wave. The small discrepancy in the iterative scheme resembles a feed back control scheme.

The main difficulties with this iterative scheme are related to the initial profile calculation, i.e. the determination of $C_{\psi}$ and $C_{f}$, and the fatal flaw if $\nabla s \cdot \nabla \varphi$ vanishes anywhere on the boundary. There is also no physical boundary constraint for advancing $\nabla_{\perp}^{2} \psi$. 


\section{ALTERNATE FORMULATION BASED ON OHM'S LAW}

A far more robust approach is based on Ohm's law, which also has the merit of a smooth transition to the reduced boundary conditions of equation (16) for axisymmetric configurations. Recall that the normal magnetic field is proportional to

$$
\mathbf{B} \cdot \nabla s=\nabla \psi \times \nabla \varphi \cdot \nabla s+\frac{1}{R} \nabla_{\perp} F \cdot \nabla s+R_{0} I \nabla \varphi \cdot \nabla s .
$$

Perfectly conducting wall implies $\mathbf{B} \cdot \nabla s=0$ so

$$
\nabla \psi \times \nabla \varphi \cdot \nabla s+\frac{1}{R} \nabla_{\perp} F \cdot \nabla s+R_{0} I \nabla \varphi \cdot \nabla s=0
$$

This will serve as an inhomogeneous Neumann boundary condition

$$
\nabla s \cdot \nabla_{\perp} F=-R_{0} R I \nabla \varphi \cdot \nabla s
$$

for solving

$$
\nabla_{\perp}^{2} F=-\tilde{I}^{\prime} / R
$$

after imposing $\psi$ a constant on the surface, for example,

$$
\psi=0
$$

which preserves half of the constraints for the axisymmetric case.

The boundary conditions for $\tilde{I}$ and $\Delta^{\star} \psi$ are provided by $\mathbf{E} \times \nabla s=0$ in the case of resistive MHD, albeit they have to be implemented iteratively, which is equivalent to the feedback control of flux leakage through a resistive wall. The reason to advance $\Delta^{\star} \psi$ instead of other variants of $\nabla_{\perp}^{2} \psi$ will become clear later. Recall the Ohm's law for a resistive plasma,

$$
\mathbf{E}+\mathbf{v} \times \mathbf{B}=\eta \mathbf{J} .
$$

Apply $\nabla s \times$ on the Ohm's law, one has

$$
\nabla s \times \mathbf{E}+\nabla s \times(\mathbf{v} \times \mathbf{B})=\eta \nabla s \times \mathbf{J} .
$$

For a perfectly conducting wall, 


$$
\begin{gathered}
\nabla s \times \mathbf{E}=0 \\
\nabla s \cdot \mathbf{v}=0 \\
\nabla s \cdot \mathbf{B}=0 .
\end{gathered}
$$

This implies

$$
\nabla s \times \mathbf{J}=0 .
$$

The boundary conditions for $\tilde{I}$ and $\Delta^{*} \psi$ are obtained by dotting $\nabla s \times \mathbf{J}$ with $\nabla \varphi$ and $\nabla \varphi \times \nabla$ s. Recall that the displacement current is ignored in the usual MHD approximation, so

$$
\mathbf{J}=\nabla \times \mathbf{B}=J_{\varphi} \hat{\varphi}+\nabla \tilde{I} \times \nabla \varphi-\frac{1}{R} \nabla_{\perp} F^{\prime} \times \nabla \varphi+\frac{1}{R^{2}} \nabla_{\perp} \psi^{\prime}
$$

Here a prime denotes toroidal derivative, for example, $F^{\prime}=\partial F / \partial \varphi$. The toroidal current $J_{\varphi}$ is related to $\psi$ and $F$ via

$$
R J_{\varphi}=-\Delta^{\star} \psi-\frac{1}{R} \frac{\partial F}{\partial Z}
$$

The tangential current is

$$
\begin{aligned}
\nabla s \times \mathbf{J}= & R J_{\varphi} \nabla s \times \nabla \varphi+(\nabla s \cdot \nabla \varphi) \nabla_{\perp} \tilde{I}-\left(\nabla s \cdot \nabla_{\perp} \tilde{I}\right) \nabla \varphi \\
& -\frac{1}{R}(\nabla s \cdot \nabla \varphi) \nabla_{\perp} F^{\prime}+\frac{1}{R}\left(\nabla s \cdot \nabla F^{\prime}\right) \nabla \varphi+\frac{1}{R^{2}} \nabla s \times \nabla_{\perp} \psi^{\prime}
\end{aligned}
$$

Hence

$$
\begin{aligned}
\nabla s \times \mathbf{J} \cdot \nabla \varphi= & -\frac{1}{R^{2}} \nabla s \cdot \nabla_{\perp} \tilde{I}+\frac{1}{R^{3}} \nabla_{s} \cdot \nabla_{\perp} F^{\prime}+\frac{1}{R^{2}} \nabla_{\perp} \psi^{\prime} \cdot(\nabla \varphi \times \nabla s) \\
\nabla s \times \mathbf{J} \cdot(\nabla \varphi \times \nabla s)= & -R J_{\varphi}(\nabla s \times \nabla \varphi)^{2}+(\nabla s \cdot \nabla \varphi) \nabla_{\perp} \tilde{I} \cdot(\nabla \varphi \times \nabla s) \\
& -\frac{1}{R}(\nabla s \cdot \nabla \varphi) \nabla_{\perp} F^{\prime} \cdot(\nabla \varphi \times \nabla s)
\end{aligned}
$$

$\nabla s \times \mathbf{J} \cdot \nabla \varphi=0$ gives an inhomogeneous Neumann boundary condition for $\tilde{I}$

$$
\nabla s \cdot \nabla_{\perp} \tilde{I}=\frac{1}{R} \nabla s \cdot \nabla_{\perp} F^{\prime}+\nabla_{\perp} \psi^{\prime} \cdot(\nabla \varphi \times \nabla s)
$$

$\nabla s \times \mathbf{J} \cdot(\nabla \varphi \times \nabla s)=0$ provides an inhomogeneous Dirichlet boundary condition for $\Delta^{*} \psi$ which is related to $R J_{\varphi}$ by $\Delta^{*} \psi=-R J_{\varphi}-\frac{1}{R} \frac{\partial F}{\partial Z}$ : 


$$
\begin{aligned}
\Delta^{*} \psi= & -\frac{1}{R} \frac{\partial F}{\partial Z}-\frac{1}{(\nabla s \times \nabla \varphi)^{2}}\left[(\nabla s \cdot \nabla \varphi) \nabla_{\perp} \tilde{I} \cdot(\nabla \varphi \times \nabla s)\right. \\
& \left.-\frac{1}{R}(\nabla s \cdot \nabla \varphi) \nabla_{\perp} F^{\prime} \cdot(\nabla \varphi \times \nabla s)\right] .
\end{aligned}
$$

Equation (23) is just of the right form upon examining the time evolution of $\Delta^{\star} \psi$,

$$
\frac{\partial}{\partial t} \Delta^{\star} \psi=\text { lower order terms }+\Delta^{\star}\left[\eta\left(\Delta^{\star} \psi+\frac{1}{R} \frac{\partial F}{\partial Z}\right)\right] \text {. }
$$

The exact boundary condition requirement of this equation is satisfied by a rearrangement of equation (23),

$$
\begin{aligned}
\Delta^{*} \psi+\frac{1}{R} \frac{\partial F}{\partial Z}= & -\frac{1}{(\nabla s \times \nabla \varphi)^{2}}\left[(\nabla s \cdot \nabla \varphi) \nabla_{\perp} \tilde{I} \cdot(\nabla \varphi \times \nabla s)\right. \\
& \left.-\frac{1}{R}(\nabla s \cdot \nabla \varphi) \nabla_{\perp} F^{\prime} \cdot(\nabla \varphi \times \nabla s)\right]
\end{aligned}
$$

In the limit of axisymmetry $(\nabla s \cdot \nabla \varphi=0)$, the general boundary condition, equations $(17,18,22,24)$, recovers the reduced ideal boundary condition discussed in section III using Faraday's law, and supplements two additional boundary conditions, from first principle, to the solution of $\tilde{I}$ and $\Delta^{\star} \psi$.

In conclusion, a complete set of physics-based boundary conditions are in place to follow the magnetic evolution in a general toroidal geometry with a perfectly conducting wall [6].

\section{ACKNOWLEDGMENTS}

Useful discussions with Wonchull Park and Hank Strauss on MH3D are gratefully acknowledged. This work was supported by U.S. Department of Energy. 


\section{REFERENCES}

[1] M. Renardy and R. C. Rogers, An introduction to partial differential equations (SpringerVerlag, New York, 1993).

[2] W. Park, E.V. Belova, G. Y. Fu, X.Z. Tang, H. R. Strauss, L.E. Sugiyama, "Plasma Simulation Studies Using Multilevel physics models", Physics of Plasma, 6 1796-1803 (1999).

[3] A. H. Glasser, C. R. Sovinec, R. A. Nebel, T. A. Gianakon, S. J. Plimpton, M. S. Chu, D. D. Schnack, and the NIMROD Team," The NIMROD code: a new approach to numerical plasma physics", Plasma Phys. Control. Fusion 41, A747 (1999).

[4] S.P. Hirshman, W.I. van Rij, and P. Merkel, "Three-dimensional free boundary calculations using a spectral Green's function method," Computer Physics Communications 43, $143-155(1986)$.

[5] L. Sugiyama and W. Park, “Notes on MH3D," unpublished.

[6] X.Z. Tang, W. Park, and H.R. Strauss, "Nonlinear resistive MHD simulation on massively parallel computers," Bulletin of the American Physical Society, Vol 44, No. 7, CP1 121 $(1999)$. 
The Princeton Plasma Physics Laboratory is operated by Princeton University under contract with the U.S. Department of Energy.

\author{
Information Services \\ Princeton Plasma Physics Laboratory \\ P.O. Box 451 \\ Princeton, NJ 08543
}

Phone: 609-243-2750

Fax: 609-243-2751

e-mail: pppl_info@pppl.gov

Internet Address: http://www.pppl.gov 